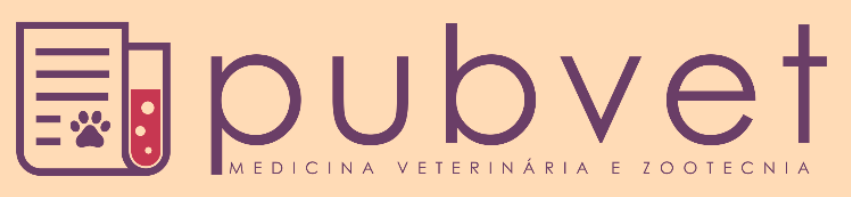

https://doi.org/10.31533/pubvet.v13n11a449.1-5

\title{
Refluxo gastroesofágico transoperatório em cães: revisão
}

\author{
Hellen Fialho Hartmann ${ }^{1 *} \bullet$, Bernardo Nascimento Antunes ${ }^{1} \bullet$, Roberta do Nascimento \\ Libardoni $^{1 \bullet}$, Luciana Gonçalves Teixeira ${ }^{2} \bullet$, Maurício Veloso Brun ${ }^{1 * *}$ \\ ${ }^{1}$ Programa de Pós-graduação em Medicina Veterinária da Universidade Federal de Santa Maria (UFSM), Santa Maria-RS Brasil. \\ ${ }^{2}$ Programa de Pós-graduação em Ciências Veterinárias da Universidade Federal do Rio Grande do Sul (UFRGS), Porto Alegre-RS Brasil. \\ *Autor para correspondência: Hellen Fialho Hartmann, pós-graduanda do PPGMV-UFSM, E-mail: hellenhartmann@gmail.com \\ **Bolsista de PQ2 do CNPq, Brasil (305076/2018-0).
}

Resumo. O refluxo gastroesofágico (RGE) transoperatório ocorre, dentre outras espécies, em humanos, cães e gatos, ocasionando danos ao esôfago devido ao baixo pH do conteúdo do trato digestório. Pode culminar em esofagite, estenose esofágica e pneumonia aspirativa. Seu diagnóstico por vezes é obtido a partir da ocorrência de regurgitação, ou seja, quando o refluxo atinge a cavidade nasal ou oral. Estudos tem sido realizado ao longo dos anos testando fármacos, biotipos corporais, procedimentos cirúrgicos ou de diagnóstico e posicionamentos que possam precipitar este evento. Neste texto é trazida uma breve revisão algumas informações sobre RGE objetivando alertar para suas importantes consequências e como preveni-las.

Palavras chave: anestesia, canino, cirurgia, refluxo

\section{Intraoperative gastroesophageal reflux in dogs: review}

Abstract. Transoperative gastroesophageal reflux (GER) occurs, among other species, in humans, dogs and cats, causing damage to the esophagus due to the low $\mathrm{pH}$ of the digestive tract content. It may culminate in esophagitis, esophageal stenosis and aspiration pneumonia. Its diagnosis is, sometimes, achieved from the occurrence of regurgitation, when the reflux reaches the nasal or oral cavity. Studies have been conducted over the years testing drugs, body types, surgical or diagnostic procedures and positions that may precipitate this event. We to gather in a brief review some information about GER in order to alert to its important consequences and how to prevent them.

Keywords: anesthesia, canine, reflux, surgery

\section{Reflujo gastroesofágico intraoperatorio en perros: revisión}

Resumen. El reflujo gastroesofágico (RGE) transoperatorio ocurre, entre otras especies, en humanos, perros y gatos, causando daño al esófago debido al bajo $\mathrm{pH}$ del contenido del tracto digestivo. Esta condición puede culminar en esofagitis, estenosis esofágica y neumonía por aspiración. Su diagnóstico, a veces, se realiza a partir de la aparición de regurgitación, es decir, cuando el reflujo alcanza la cavidad nasal u oral. A lo largo de los años, se han realizado estudios investigando medicamentos, biotipos corporales, procedimientos quirúrgicos o de diagnóstico y posiciones que pueden precipitar este evento. Buscamos aquí reunir en una breve revisión información sobre RGE para alertar sobre sus importantes consecuencias y cómo prevenirlas.

Palabras clave: anestesia, caninos, cirugía, reflujo 


\section{Introdução}

Refluxo gastroesofágico (RGE) é dito o conteúdo gastroduodenal que migra para o esôfago e adjacências e pode resultar numa gama de sinais (Barczinski \& Moraes-Filho, 2006). Suas sequelas associadas são causas descritas de morbidade e mortalidade tanto em humanos como cães e gatos (Zacuto et al., 2012). O RGE, juntamente com regurgitação, náusea, vômitos e íleo paralítico, são as desordens gastrointestinais mais comuns associadas a um processo de anestesia geral (Torrente et al., 2017). No período transoperatório o RGE passa despercebido a menos que atinja a cavidade oral ou nasal (Wilson et al., 2007). Quando o conteúdo gastroduodenal atinge a faringe é denominado regurgitação e pode ser aspirado pelo pulmão (Wilson et al., 2005). O RGE durante a anestesia está ainda associado a 46-65\% dos casos de estenose esofágica em cães sendo a principal causa de esofagite grave seguida de estenose (Zacuto et al., 2012). A incidência de RGE transoperatório na espécie canina varia muito conforme protocolo anestésico utilizado e procedimento realizado, mas se encontra entre 16,3 e $55 \%$, enquanto que a incidência de regurgitação durante anestesia seria de 0,5 a $10 \%$ (Wilson et al., 2007). Sem métodos diagnósticos especiais, apenas as regurgitações são percebidas clinicamente, daí a importância de se pesquisar mais sobre este evento de consequências tão graves.

\section{Etiologia}

Diferentemente da espécie humana, cuja causa mais comum de estenose esofágica é a injúria péptica ocasionada por exposição crônica da mucosa esofágica ao pH gástrico (Siersema \& Wijkerslooth, 2009), nos caninos a causa mais comum desta grave afecção é o RGE perianestésico (Lam et al., 2013). O tônus no esfíncter esofágico caudal é responsável por aumentar a pressão na junção esôfago-gástrica (Farre \& Sifrim, 2008) e mecanismos não adrenérgicos e não colinérgicos são responsáveis pelo relaxamento deste esfíncter (Kortezova et al., 1996). Anestésicos voláteis e fármacos utilizados na pré-medicação parecem interferir no relaxamento do esfíncter esofagogástrico (Wilson et al., 2005). A pressão na cárdia de cães é diminuída com o uso de isofluorano, atropina, acepromazina e xilazina (Hashim et al., 1995). Uma redução na pressão do esfíncter esofágico caudal aumenta o risco de RGE, mas não significa que ele irá ocorrer (Wilson et al., 2007). Inibidores da bomba de prótons ou agentes pró-cinéticos podem reduzir o risco de RGE (Rodríguez-Alarcón et al., 2015). O RGE ocorreu em aproximadamente 50\% dos cães que receberam morfina na pré-medicação em um estudo (Wilson et al., 2005). Cães prémedicados com medetomidina parecem apresentar um maior risco de regurgitação transoperatória do que aqueles medicados com acepromazina associada com algum opioide (García et al., 2013).

Em um estudo, $100 \%$ das cadelas submetidas à videocirurgia pré-medicadas com morfina manifestaram RGE, enquanto 30\% manifestaram quando submetidas à laparotomia sob mesmo protocolo anestésico (Hartmann et al., 2018). Isto porque fatores influenciam no RGE, de forma que uma pressão intratorácica diminuída e/ou uma pressão abdominal aumentada somado a um relaxamento da cárdia atuam mecanicamente, enquanto o tônus parassimpático vagal que afeta a cárdia e causa efeitos sobre o esvaziamento e indiretamente sobre o relaxamento esofágico é considerado um fator fisiológico (Reid, 2018).

Fatores inerentes ao paciente como tipo corporal, duração e tipo do procedimento também influenciam na incidência de RGE (Torrente et al., 2017). Animais de raças grandes parecem mais predispostos ao RGE (Lamata et al., 2012). A composição do refluxo pode influenciar a gravidade da esofagite subsequente de maneira que quanto mais ácida a secreção gástrica, maior a erosão da mucosa esofágica (Evander et al., 1987). Tal essa acidez varia conforme tempo de jejum e alimentação do animal (Sagawa et al., 2009; Savvas et al., 2016).

\section{Diagnóstico}

Os refluxos são classificados como ácidos $(\mathrm{pH}<4,0)$, fracamente ácidos $(4,0>\mathrm{pH}<7,5)$ ou não-ácidos (pH > 7,5) (Zacuto et al., 2012). E interferem nessa acidez, além do tempo de jejum e o tipo de alimentação do animal (Sagawa et al., 2009) e o uso de inibidores da bomba de prótons (Panti et al., 2009) entre outros. O refluxo ácido é geralmente oriundo do estômago e o não ácido de uma mistura entre secreção biliar e gástrica (Favarato et al., 2010); porém, ácidos biliares já foram encontrados na mucosa de esôfagos gravemente lesionados em humanos, indicando que este tipo de refluxo pode ser ainda mais grave, provavelmente devido um sinergismo tóxico entre secreções gástricas e duodenais (Nehra et al., 1999). 
O diagnóstico do RGE nos cães conscientes depende da pHmetria, da monitorização da bilirrubina, da impedância intraluminal multicanal ou da manometria. Já no período transanestésico, podemos nos valer também da endoscopia (Favarato et al., 2010). A pHmetria esofágica sozinha poderia subestimar a ocorrência de RGE já que não detecta mudanças sutis no $\mathrm{pH}$ e poderia não detectar refluxos nãoácidos, sendo ideal associa-la a impedância intraluminal multicanal (Weigt et al., 2007). A impedância intraluminal multicanal é uma técnica que mede a oposição de corrente elétrica entre dois eletrodos e, portanto, detecta o fluxo do bolo através do esôfago (Hernani et al., 2013). A manometria esofágica determina os níveis de pressão nos diferentes segmentos do esôfago, que, quando alterados, associamse à doença do refluxo gastroesofágico (Oba, 2011).

\section{Consequências}

A incidência de pneumonia aspirativa pós-anestesia resultante de refluxo gastroesofágico em cães é de $0,17 \%$ (Ovbey et al., 2014) e em humanos, de 0,014 a 0,05\% (Janda et al., 2006), sendo uma grave causa de morbidade e mortalidade anestésica (Nogueira et al., 2003).

A estenose esofágica pode ocorrer secundariamente a uma esofagite, que pode ser causada por alojamento seguido de remoção de corpo estranho (Luthi \& Neiger, 1998), ingestão de substância corrosiva (German et al., 2005), ou RGE cujo conteúdo ácido ficou em contato com a mucosa esofágica por determinado tempo, especialmente durante cirurgias (Adamama-Moraitou et al., 2002). Para que ocorra a estenose, a lesão deve atingir a camada muscular do esôfago, o que culmina em formação de tecido cicatricial (Galatos \& Raptopoulos, 1995). A importância do RGE é tamanha que uma taxa de $23 \%$ de mortalidade é associada à disfunção esofágica pós-anestesia (Wilson \& Walshaw, 2004).

\section{Cenário atual}

Foi demonstrado que, em cães, uma refeição leve $3 \mathrm{~h}$ anterior ao procedimento anestésico-cirúrgico diminuiu significantemente a incidência de RGE e manteve os pHs no trato digestivo mais elevados, dificultando assim, a esofagite e/ou estenose, no caso de ocorrência de RGE (Savvas et al., 2016).

Já foram realizados estudos utilizando fármacos como metoclopramida (Wilson et al., 2006), meperidina (Wilson et al., 2007), inibidores da bomba de prótons (Panti et al., 2009), esomeprazole e cisaprida (Zacuto et al., 2012) morfina, tramadol e metadona (Hartmann et al., 2017) a fim de verificar a influência sobre o refluxo ou se algum poderia, de fato, reduzir ou zerar a incidência de RGE. O uso de esomeprazole associado à cisaprida reduziu a incidência de RGE em cães anestesiados ao passo que o esomeprazole sozinho foi responsável pelo aumento do $\mathrm{pH}$, não causando esofagite ou estenose quando da ocorrência de RGE (Zacuto et al., 2012).

O RGE já foi associado com maior incidência em OVH videocirúrgicas com dois portais de acesso em comparação a convencionais (Hartmann et al., 2018). Cães braquicefálicos já foram, sem sucesso, incluídos no grupo de risco de apresentar RGE transanestésico (Shaver et al., 2017). Já RodríguezAlarcón et al. (2015) observaram que cães da raça poodle, posicionamento em Trendelemburg ou decúbito lateral direito, cirurgias ortopédicas utilizando atropina e morfina na pré-medicação e o uso de propofol como agente indutor anestésico foram fatores de risco para RGE transoperatório. García et al. (2013) verificaram em seu estudo retrospectivo que cães maiores, ASA três ou mais, submetidos a cirurgia abdominal ou exames de imagem sob anestesia incluem-se no grupo de risco para potencial RGE visível.

\section{Considerações finais}

O RGE é um evento importante que pode culminar em sérias consequências pós-anestésicas como estenose esofágica e pneumonia aspirativa, podendo, portanto, elevar os índices de morbidade e mortalidade pós-anestésicas. Não há predileção sexual ou racial, embora animais de porte maior pareçam ser mais acometidos. A escolha dos fármacos pré-anestésicos, o posicionamento na mesa cirúrgica e o tipo de procedimento a ser realizado podem tonar o paciente mais predisposto ao evento. Ações simples são válidas para reduzir os riscos de RGE no transoperatório, tais como realizar uma boa anamnese para detectar fatores de risco como doenças gastrointestinais pré-existentes, não fazer jejum prolongado e utilizar racionalmente inibidores da bomba de prótons ou pró-cinéticos. Mais estudos se 
fazem necessários para definir protocolos anestésicos e cirúrgicos que possam inibir totalmente a ocorrência de RGE transoperatório.

\section{Agradecimentos}

À Coordenação de Aperfeiçoamento de Pessoal de Nível Superior (CAPES) e ao Conselho Nacional de Desenvolvimento Científico e Tecnológico (CNPq).

\section{Referências bibliográficas}

Adamama-Moraitou, K. K., Rallis, T. S., Prassinos, N. N. \& Galatos, A. D. (2002). Benign esophageal stricture in the dog and cat: a retrospective study of 20 cases. Canadian Journal of Veterinary Research, 66(1):5559.

Barczinski, T. \& Moraes-Filho, J. P. P. (2006). Doença do refluxo gastroesofágico na mulher. Revista Brasileira de Medicina, 63(12):160-168.

Evander, A., Little, A. G., Riddell, R. H., Walther, B. \& Skinner, D. B. (1987). Composition of the refluxed material determines the degree of reflux esophagitis in the dog. Gastroenterology, 93280-286.

Farre, R. \& Sifrim, D. (2008). Regulation of basal tone, relaxation and contraction of the lower oesophageal sphincter. Relevance to drug discovery for oesophageal disorders. British Journal of Pharmacology, 153(5):858-869.

Favarato, E. S., Souza, M. V. \& Costa, P. R. S. (2010). Refluxo gastroesofágico em cães anestesiados: fisiopatologia, clínica, diagnóstico e terapêutica. Ciência Rural, 40(11):2427-2434.

Galatos, A. D. \& Raptopoulos, D. (1995). Gastro-oesophageal reflux during anaesthesia in the dog: the effect of preoperative fasting and premedication. The Veterinary Record, 137(19):479-483.

García, C. M., Pinchbeck, G. L., Dugdale, A. \& Senior, J. M. (2013). Retrospective study of the risk factors and prevalence of regurgitation in dogs undergoing general anaesthesia. The Open Veterinary Science Journal, $7(1)$.

German, A. J., Cannon, M. J., Dye, C., Booth, M. J., Pearson, G. R., Reay, C. A. \& Gruffydd-Jones, T. J. (2005). Oesophageal strictures in cats associated with doxycycline therapy. Journal of Feline Medicine and Surgery, 7(1):33-41.

Hartmann, H. F., Feranti, J. P., Oliveira, M. T., Linhares, M. T., Correa, L. F. D., Coradini, G. P. \& Brun, M. V. (2018). Refluxo gastroesofágico em cadelas submetidas à ovário-histerectomia convencional ou videoassistida. Arquivo Brasileiro de Medecina Veterinária e Zootecnia, 70(1):101-108.

Hartmann, H. F., Feranti, J. P. S., Oliveira, M. T., Linhares, M. T., Correa, L. F. D., Coradini, G. P. \& Brun, M. V. (2017). Refluxo gastroesofágico em cadelas durante ovário-histerectomia convencional submetidas a diferentes medicações pré-anestésicas. Arquivo Brasileiro de Medicina Veterinária e Zootecnia, 69(5):1231-1235.

Hashim, M. A., Waterman, A. E. \& Pearson, H. (1995). A comparison of the effects of halothane and isoflurane in combination with nitrous oxide on lower oesophageal sphincter pressure and barrier pressure in anaesthetised dogs. The Veterinary Record, 137(26):658-661.

Hernani, M. M. T., Calatayud, G. A. \& Sanchéz, C. S. (2013). Impedanciometría intraluminal multicanal esofágica: indicaciones y técnica. Anales de Pediatría Continuada, 11(2):110-116.

Janda, M., Scheeren, T. W. L. \& Nöldge-Schomburg, G. F. E. (2006). Management of pulmonary aspiration. Best practice \& Research Clinical Anaesthesiology, 20(3):409-427.

Kortezova, N., Mizhorkova, Z., Milusheva, E., Varga, G., Vizi, E. S. \& Papasova, M. (1996). Non-adrenergic non-cholinergic neuron stimulation in the cat lower esophageal sphincter. European Journal of Pharmacology, 304(1-3):109-115.

Lam, N., Weisse, C., Berent, A., Kaae, J., Murphy, S., Radlinsky, M., . . Gingerich, K. (2013). Esophageal stenting for treatment of refractory benign esophageal strictures in dogs. Journal of Veterinary Internal Medicine, 27(5):1064-1070.

Lamata, C., Loughton, V., Jones, M., Alibhai, H., Armitage-Chan, E., Walsh, K. \& Brodbelt, D. (2012). The risk of passive regurgitation during general anaesthesia in a population of referred dogs in the UK. Veterinary Anaesthesia and Analgesia, 39266-274. 
Luthi, C. \& Neiger, R. (1998). Esophageal foreign bodies in dogs: 51 cases (1992-1997). The European Journal of Comparative Gastroenterology, 3(2):7-11.

Nehra, D., Howell, P., Williams, C. P., Pye, J. K. \& Beynon, J. (1999). Toxic bile acids in gastrooesophageal reflux disease: influence of gastric acidity. Journal of the British Society of Gastroenterology, 44598-602.

Nogueira, L. C., Cortopassi, S. R. G., Intelizano, T. R. \& Souza, M. S. B. (2003). Efeitos do jejum alimentar pré-cirúrgico sobre a glicemia e o período de recuperação anestésica em cães. Brazilian Journal of Veterinary Research and Animal Science, 4020-25.

Oba, J. (2011). Recomendações para diagnóstico e tratamento do refluxo gastroesofágico em pediatria. Educação Continuada em Saúde, 9(2):57-61.

Ovbey, D. H., Wilson, D. V., Bednarski, R. M., Hauptman, J. G., Stanley, B. J., Radlinsky, M. G. \& Rezende, M. L. (2014). Prevalence and risk factors for canine post-anesthetic aspiration pneumonia (1999-2009): a multicenter study. Veterinary Anaesthesia and Analgesia, 41(2):127-136.

Panti, A., Bennett, R. C., Corletto, F., Brearley, J., Jeffrey, N. \& Mellanby, R. J. (2009). The effect of omeprazole on oesophageal pH in dogs during anaesthesia. Journal of Small Animal Practice, 50540-544.

Reid, K. (2018). Perioperative gastrointestinal reflux in dogs. In Practice, 40(9):370-382.

Rodríguez-Alarcón, C. A., Beristain-Ruiz, D. M., Rivera-Barreno, R., Díaz, G., Usón-Casaús, J. M., GarcíaHerrera, R. \& Pérez-Merino, E. M. (2015). Gastroesophageal reflux in anesthetized dogs: a review. Revista Colombiana de Ciencias Pecuarias, 28(2):144-155.

Sagawa, K., Li, F., Liese, R. \& Sutton, S. C. (2009). Journal of Pharmaceutical Sciences, 98(7):2494-2500.

Savvas, I., Raptopoulos, D. \& Rallis, T. (2016). A "light meal" three hours preoperatively decreases the incidence of gastro-esophageal reflux in dogs. Journal of the American Animal Hospital Association, 52(6):357-363.

Shaver, S. L., Barbur, L. A., Jimenez, D. A., Brainard, B. M., Cornell, K. K., Radlinsky, M. G. \& Schmiedt, C. W. (2017). Evaluation of gastroesophageal reflux in anesthetized dogs with brachycephalic syndrome. Journal of the American Animal Hospital Association, 53(1):24-31.

Siersema, P. D. \& Wijkerslooth, L. R. H. (2009). Dilation of refractory benign esophageal strictures. Gastrointestinal Endoscopy, 70(5):1000-1012.

Torrente, C., Vigueras, I., Manzanilla, E. G., Villaverde, C., Fresno, L., Carvajal, B., . . Costa-Farré, C. (2017). Prevalence of and risk factors for intraoperative gastroesophageal reflux and postanesthetic vomiting and diarrhea in dogs undergoing general anesthesia. Journal of Veterinary Emergency and Critical Care, 27(4):397-408.

Weigt, J., Mönkemüller, K., Peitz, U. \& Malfertheiner, P. (2007). Multichannel intraluminal impedance and pH-metry for investigation of symptomatic gastroesophageal reflux disease. Digestive Diseases, 25(3):179182.

Wilson, D. V., Evans, A. T. \& Mauer, W. A. (2006). Influence of metoclopramide on gastroesophageal reflux in anesthethized dogs. American Journal of Veterinary Research, 67(1):26-31.

Wilson, D. V., Evans, A. T. \& Miller, R. (2005). Effects of preanesthetic administration of morphine on gastroesophageal reflux and regurgitation during anesthesia in dogs. American Journal of Veterinary Research, 66(3):386-390.

Wilson, D. V., Tom Evans, A. \& Mauer, W. A. (2007). Pre-anesthetic meperidine: associated vomiting and gastroesophageal reflux during the subsequent anesthetic in dogs. Veterinary Anaesthesia and Analgesia, 34(1):15-22.

Wilson, D. V. \& Walshaw, R. (2004). Postanesthetic esophageal dysfunction in 13 dogs. Journal of the American Animal Hospital Association, 40(6):455-460.

Zacuto, A. C., Marks, S. L., Osborn, J., Douthitt, K. L., Hollingshead, K. L., Hayashi, K. \& Belafsky, P. C. (2012). The influence of esomeprazole and cisapride on gastroesophageal reflux during anesthesia in dogs. Journal of Veterinary Internal Medicine, 26(3):518-525.

Recebido: 16 de outubro, 2019.

Aprovado: 23 de novembro, 2019.

Publicado: 12 de dezembro, 2019
Licenciamento: Este artigo é publicado na modalidade acesso aberto sob a licença Creative Commons Atribuição 4.0 (CC-BY 4.0), a qual permite uso irrestrito, distribuição, reprodução em qualquer meio, desde que o autor e a fonte sejam devidamente creditados. 